\title{
Emotional Interaction Agents in Intelligent Tutoring Systems ${ }^{*}$
}

\author{
SUN Yu, LI Zhiping", XIA Yaowen \\ School of Information Science and Technology \\ Yunnan Normal University \\ Kunming, Yunnan Province, China \\ sunyu_km@hotmail.com; ynnulzp@foxmail.com; xiayaowen@ynnu.edu.cn
}

\begin{abstract}
A model of emotional interaction agents in intelligent tutoring systems is presented in this paper, and the functionalities of the key components of the agents are described. To improve the emotional interaction between learners and the system, a kind of emotional interaction agents which can deduce users' emotional statues, provide helps needed, and mark emotional difficulty of the learned pedagogical units, is introduced and discussed in detail in order to improve pedagogical effects.
\end{abstract}

Keywords - intelligent tutoring systems; emotional interaction agents; emotion lacking.

\section{INTRODUCTION}

Intelligent Tutoring Systems (ITSs) is a branch of artificial intelligence and forms an advanced generation of Computer Aided Instruction systems [1,2]. Besides artificial intelligence, the research of ITS also involves many subjects such as computer science, cognitive science, pedagogy, psychology, behaviour science, and so on. The ultimate purpose of ITS is to imitate excellent teachers and to teach like a real human beings.

The key feature of intelligent tutoring systems is the ability to provide a user self-adapted presentation of the teaching material [3] by using artificial intelligence methods to represent the pedagogical decisions and the information about each user. The emergence of the WWW has increased the applications of such systems [4,5].

Although some tutoring systems have correct information and appropriate teaching strategies, a learner might still lack the motivation to complete a learning process. The main reason is that these systems apply the same approach to all learners and do not consider users' different knowledge levels, learning styles, and demands.

In traditional classroom education, teachers communicate with students face to face. They know the emotional situations of students and can give students some feedbacks or helps at once. However, in intelligent tutoring systems, there is no this kind of two-way interaction between students and teachers. Hence, when student encounters troubles during the processes of learning, they cannot talk to the system like that of talking with teacher in classroom. Thus, they will lose confidence or even may quit their learning.

Even if some systems take a user's characteristics (e.g. knowledge levels, learning styles, etc.) and needs into account, how to choose the right learning material to the right user is especially important in order to reach the desired teaching effects.

Hence, we introduce the emotional interaction agents. Based on the knowledge about a user, an emotional interaction agent can deduce users' emotional statues, provide helps needed, and mark emotional difficulty of the learned pedagogical units. Furthermore we use ontologies and pedagogical units with multi-media materials for domain knowledge representation to improve the sharing and reusing of materials.

The paper is organized as follows. Section II discusses the problem of emotional lacking occurring in intelligent tutoring systems. Section III introduces the ontology-based domain knowledge representation and pedagogical units of the system. Section IV gives an overview of the architecture of the emotional interaction agents in our intelligent tutoring systems, and then discusses the four modules of emotional interaction agents in detail. Finally, section V concludes the paper with conclusion and future works.

\section{THE Problem of EMOTION LACKING}

In this section, we first introduce the motion of emotional communication, and discuss the problem of emotion lacking in intelligent system through the comparison between the tradition classroom teaching and distance learning.

In Education, emotional communication means that the interaction of expressions, emotions and evaluation between teachers and students. In his book named "Emotional

\footnotetext{
* The work is supported by the National NSF of China (No. 60903131), Key Laboratory of Nationality Educational Informalization (Yunnan Normal University), Ministry of Education(No. EIN2011B002,EIN2011B004), Key Project of Science and Technology Research of Education Ministry (No. 210210), the NSF of Yunnan Province (No.2004F0017Q, 2005F0022Q, 2009ZC0052M, 2012FB144), the Program for Innovative Research Team (in Science and Technology) in University of Yunnan Province and the Reserve Force of academic technology leader in Yunnan Province.

\# Corresponding Author
} 
Intelligence", American psychologist Goleman pointed out: Emotional Intelligence is a kind of important symbol of human intelligence[6].At the same time, the purpose of course construction should emphasize on knowledge and technique, procedure and methodology, and emotion and attitude. Compared with the learning of knowledge and technique, emotion and attitude guide the learning motivations and preferences of students.

In traditional classroom education, teachers can know the emotional situations of students and can give students feedbacks or helps accordingly in time. However, in intelligent tutoring systems, there are barely this kind of two-way interaction between students and teachers.

In intelligent tutoring systems, although there are rich multimedia materials, but the systems can hardly act like a real teacher. Students cannot interact with these systems face to face like the way teachers communicate with students in classrooms. Learners are considered as "listeners"[7]. It is difficult for teacher to do emotional communication with students and to make some feedback according to this kind of interaction. Thus, if the learning materials or learning methods are not fit for a student, he would like to lose confidence and to feel boring. If this situation goes on without the help of teachers, the student may quit learning. All this will lead to a serious emotionlacking or emotion-missing problem in intelligent tutoring system.

In traditional classroom instruction, teachers can figure out what difficulties that a student faces with based on his emotional situation. Thus, they can provide individualized assistance for the student. However, in intelligent tutoring systems, the lacking of emotional interaction will lead to the problem that students cannot get help when they need in time. Thus, students' learning interests and motivation will be affected, and students' learning effects will be reduced ultimately.

In order to solve the problem of emotion-lacking or emotion-missing in some degree, we introduce emotional interaction agents in our intelligent tutoring system.

\section{PEDAGOGICAL UNITS}

In this section, we discuss the representation of domain knowledge based on ontologies.

The introduction of ontologies can overcome the general problem in existing teaching systems [8]. At the same time, web introduce rich multimedia teaching materials by texts, images, audios, videos, examples and so on to make the dynamic interaction between systems and users and between users available [9]. Besides effective communication, dynamic interaction improves the abilities of problem solving and learning.

Hence, we construct the representation frame of domain knowledge based on ontologies to improve the sharing and reusing of teaching materials; and we fuse the rich teaching materials provided by web into the frame.

In the construction of our ontology-based knowledge base, three-level ontology-based knowledge representation structure showed in figure 1 is proposed.

The first two levels are represented by a separate ontology, that is, there are two kinds of ontologies: subject ontology and knowledge concept ontology and the third level is pedagogical units level.

And two kinds of relations are introduced to express the relationships between subject ontology and knowledge concept ontology; and between knowledge concept ontology and pedagogical units.

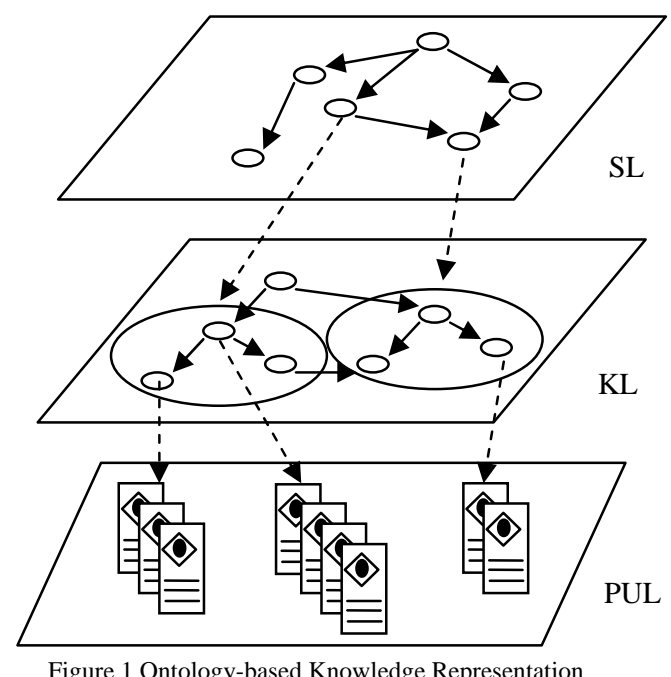

Then we introduce the above three-level knowledge representation structure showed in figure 1.

- subject ontology level (SL): the subject content of learning and the class hierarchy among these contents form the main structure of the ontology.

- knowledge concept ontology level (KL): the knowledge concepts and the class hierarchy among these conepts form the main structure of the ontology; furthermore, the relations such as part-of, prerequisite, etc., are also considered.

- pedagogical units level (PUL): includes the set of pedagogical units, each unit corresponds to some knowledge concept in the knowledge concept ontology level. For a pedagogical unit, there is one and at most one knowledge concept in the second level that corresponds to it, however, for a knowledge concepe, there maybe more than one pedagogical unit corresponding to it.

A pedagogical unit consists of multimedia materials used to teach a knowledge concept. These multimedia materials can be text materials, image materials, example materials, exercise materials, video materials, audio materials, and so on.

From figure 1, we can see that a subject corresponds to a of knowledge concepts used to describe the subject; and a 
knowledge concept corresponds to plenty of pedagogical unit used to teach the concept.

The multimedia materials organized by some teaching strategies forms a pedagogical unit.

Due to the differences among uses' teaching strategy preferences, learning styles, memory and knowledge levels, etc. and the richness of materials, a same knowledge concept may correspond to several pedagogical units.

We don't consider the formation of these pedagogical units in the third level of our knowledge base in this paper. We just assume that there is a pedagogical units level consisiting of pedagogical units.

\section{EMOTIONAL INTERACTION AGENTS}

In this section, we first introduce knowledge maps, then discuss them in intelligent tutoring systems.

Emotions are an essential part of human life; they influence how we think, adapt, learn, behave, and how humans communicate with others. It is clear that without the preferences reflected by positive and negative effects, our experiences would be a neutral gray.

Based on the knowledge about users and ontology-based knowledge bases, an emotional interaction agent tries to emitate the emotional communication task of tradictional classroom teachers in order to find problems and difficulties encountered by users during the process of learning. Hence, it must have the intelligence of expression recognition, emotion analysis and process, pedagogical unit emotional notation, and help provided.

As showed in figure 2, the model of emotional interaction agents consists of three modules: expression recognition module, emotion analysis and process module, pedagogical unit emotional notation module and help provided module.

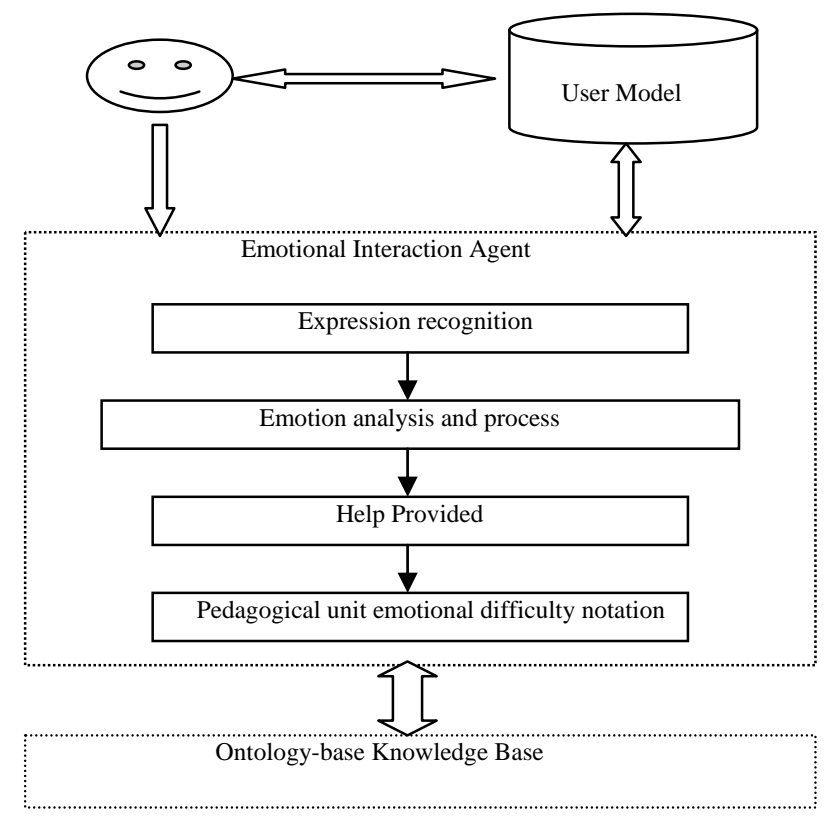

Figure 2. Emotional Interaction Agents
One of the main tasks of emotional interaction agents is to intimate teachers in traditional classroom to perform emotional communication with students. Through a camera in front of a student, emotional pedagogical agents collect information getting from the camera, then deduce the student's emotional situation based on the analysis of this information. Finally, from the emotional statues of students observed in advance, the agents can recognize the problems that students are encountered with, and provide a solution for students.

For an emotional interaction agent, the procedure of recognizing, studying and solving a student's problems can be described by the following four modules.

- Expression recognition.

By using cameras or some other methods, emotional interaction agents acquire students' expression data, and then derive useful expression information form this data.

- Emotion analysis and process.

Through the analysis of the expression information getting from the former step, the agents obtain users' emotional status according to some classification standard of emotions.

- Help provided.

According to a student's emotional statu and previous difficulty mark of the pedagogical unit, emotional interaction agents may comprimize the student or give some help.

- Pedagogical unit emotional difficulty notation.

According to users' emotional status obtianed from emotion analysis and process step, the agents put emotional difficulty mark on the pedagogical unit that is being learning.

Then we discuss the above four modules of emotional interaction agent in detail.

Since there is no standard definition for emotions [10] and there are a lot of different definitions in the literature, we did not limit the definition and the representation method of emotions. For simplicity, we divide emotions into three types: positive emotion (such as joyful, proud and elated), normal emotion and negative emtion (such as angry, sad, fearful, ashamed and desperate).

When learners first come to our intelligent tutoring system, a standard picture is taken and stored in user expression databse. From that picture, the positions and shapes of the user's eyes, nose, eyebrows and mouth are analyzed in detail.

In expression recognition module, information produced by analyzing data from sensors like cameras may include

- kinematic and dynamic quantities (positions, speeds, or energies);

- recognized symbolic gestures, the degree to which learners (or parts of their bodies) remain in tempo; 
- the smoothness of the learners' movement.

From these sensors, we can obtain reactive and rational inputs, which may include descriptions of kinematic and dynamic quantities, gestures, and movement features. Different gestures may produce different emotional stimuli.

However, in this paper, we only consider face characteristics getting from different sensors. After the analysis of these face characteristics, our emotional interaction agents recognize users' face expression.

In emotion analysis and process module, two databases are used. One is expression database and another is emotion database.

Accoring to the recognized expression, emotional interaction agents use the emotion analysis and process module to obtain users' current emotion based on expression database, emotion database and the relationship between expression databse and emotion database.

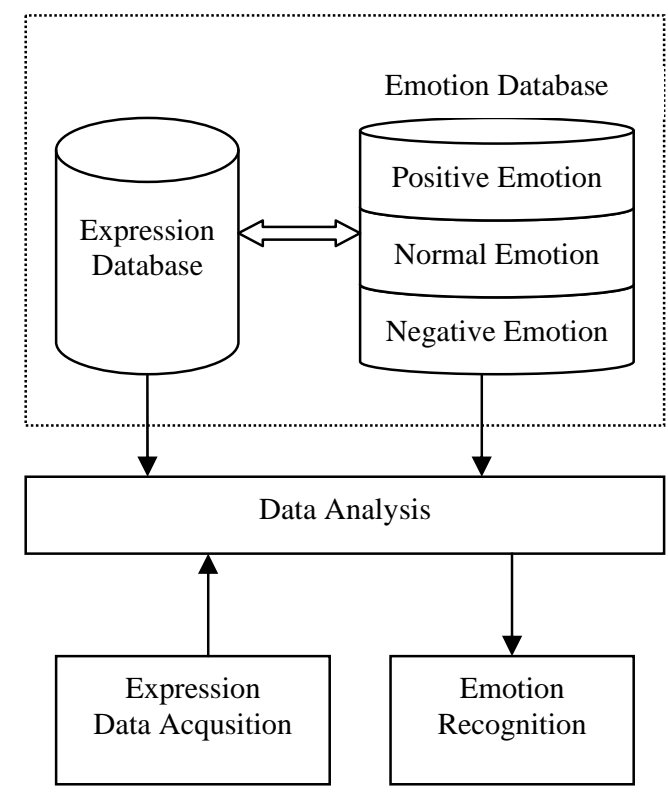

Figure 3 Expression Analysis and Process

We assume that there are three kinds of emotions: positive emotion, normal emotion or negtive emotion in our emotion database, and every kind of emotion is described by a set of emotion masterplates. After users' emotion has been recognized, we classifies it into positive emotion, normal emotion or negative emotion based on these emotion masterplates.

In the help provided module, according to a student's emotional statu and previous difficulty mark of the pedagogical unit, emotional interaction agents may comprimize the student or give some help.

Finally, in pedagogical unit emotional difficulty notation module, according to users' emotional status obtianed from emotion analysis and process module, the agents put emotional difficulty mark on the learned pedagogical unit.

\section{CONCLUSION AND FUTURE WORKS}

A model of emotional interaction agents in intelligent tutoring systems is presented with the following properties:

$>$ After the discussion of emotion lacking problem, we introduce emotional interaction agents in order to improve the pedagogical effects of intelligent tutoring systems.

$>$ The system represents domain knowledge based on ontologies to improve the sharing and reusing of domain knowledge. Furthermore, multi-media teaching materials are utilized to meet users different preferences.

In a whole, the lacking of face-to-face emotional communication in web-based intelligent tutoring systems reduces users' learning motivation. Hence, emotional interaction agents are disccussed in this paper in detail.

As future directions, we intend to develop the cognitive structure of emotional interaction agents. Furthermore, the generation of ontologies that describe knowledge bases and algebra operation of these ontologies are also our future research works.

\section{REFERENCES}

[1] J.M. Fritz, Provides Intelligence in Web-based Tutors. North American Web Developers Conference, October 1998.

[2] G. Katerina, The design of a 'motivating' intelligent assessment system, In Proceedings of Lecture Note in Computer Science 1839, pages 208-218, 2002.

[3] Vassileva, J.: Dynamic Courseware Generation. In Journal of Computing and Information Technology, 5: 87-102,1997.

[4] R. Reddy: Three open problems in AI. JACM 50(1): 83-86, 2003.

[5] M. Specht, R. Oppermann, ATS-Adaptive Teaching System: a WWWbased ITS, In U. Timm (Eds.), Proceedings of Workshop Adaptivität und Benutzer modellierung in Interaktiven Software systemen: ABIS 1998.

[6] Goleman D. Emotional Intelligence [M]. Bantam Books, 1995: 23-26.

Jiahua Zhang etc. Network course content to present the form of contrast experiment research[J]. modern educational technology, 2009.12

[7] M. Specht, R. Oppermann, ATS-Adaptive Teaching System: a WWWbased ITS [C], In U. Timm (Eds.), Proceedings of Workshop Adaptivität und Benutzer modellierung in Interaktiven Software systemen: ABIS 1998.

[8] P. Brusilovsky, Adaptive and Intelligent Technologies for Web-based Education [M]. In: Rollinger, C., Peylo, C. (eds.) Kunstliche Intelligenz, Special Issue on Intelligent Systems and Teleteaching, 1999.

[9] P. Kleinginna and A. Kleinginna. A categorized list of emotion definitions, with suggestions for a consensual definition. Motivation and Emotion, 5:345-379, 1981.

[10]P. Brusilovsky, Adaptive and Intelligent Technologies for Web-based Education. In: Rollinger, C., Peylo, C. (eds.) Kunstliche Intelligenz, Special Issue on Intelligent Systems and Teleteaching, 1999.

[11]N.Manouselis \& D.Sampson: Dynamic Knowledge Route Selection for Abstract- 